\title{
Non peptidic small molecular inhibitors of the p53-MDM2 interaction
}

\author{
Chiragkumar J. Gohil ${ }^{1 *}$, Malleshappa N. Noolvi² \\ ${ }^{1}$ Ph.D Scholar, ${ }^{2}$ Professor and Principal, ${ }^{2}$ Dept. of Pharmaceutical Chemistry, ${ }^{1}$ Discipline of Pharmacy, Gujarat \\ Technological University, Ahmedabad, Gujarat, ${ }^{2}$ Shree Dhanvantary Pharmacy College, Kim(E), Surat, Gujarat, India \\ Corresponding Author: Chiragkumar J. Gohil \\ Email: gohil2711@gmail.com
}

\begin{abstract}
Cancer is a tumorous disease, which involves the unwanted cell growth and cell division. The imbalance or inactivity of the apoptosis in the body is responsible for the occurrence of tumour and cancer. This apoptosis is regulated by the p53 protein, which is tumour suppressor protein. In the cancer cells, this p53 has been inhibited by the MDM2 protein. MDM2 interact with the p53 and make it inactive. This p53-MDM2 interaction is responsible for the cancer genesis. If we target this interaction, then we can initiate the apoptosis in the cancer cells by making the free $\mathrm{p} 53$ protein. There are many strategies to inhibit this p53-MDM2 interaction. Among them non-peptidic small molecule inhibitors are the convenient approach. Small molecule inhibitors have a three pocket binding, so they bind with p53 binding pocket ( $\operatorname{Trp} 23$, Leu 26 and Phe 19), present in the MDM2 protein. That is how it spares the p53 protein and makes it available in the cancer cells. Hence, small molecule inhibitors successfully inhibit the p53-MDM2 interaction and can initiate the apoptosis in the cancer cells, which are having the un-mutated p53 protein. They can't inhibit this interaction in the cells which contains the mutated or deleted p53 protein. Hence, this limitation must be addressed. Many of the small molecular MDM2 inhibitors have been successfully entered into the clinical trials and they are performing well. The clinical data indicate that the small molecular MDM2 inhibitors are having very low toxicity to the normal cells. And they are non-genotoxic so they are near to nontoxic to the normal cells. But none of the any small molecule MDM2 inhibitor has been enters into the market yet. So till then, it has required advancement and research to make more selective and specific for the cancer cells over the normal cells.
\end{abstract}

Keywords: Cancer, Apoptosis, p53 protein, MDM2 protein, p53-MDM2 interaction, Three pocket binding, Small molecule p53-MDM2 interaction inhibitors.

\section{Introduction}

Even though the medical science has been advanced a lot, there are certain diseases, which are still challenge for the medical science. Cancer is one of these disease. The uncontrolled cell growth causes cancer. ${ }^{1}$ Cancer is characterized by the appearance of Tumor, which is an abnormal mass of the cells. Activation of the Oncogenes in the body is responsible for the cancer. Mainly there are two types of tumors, Malignant Tumor and Benign Tumor. Tumors can invade the vital organs of the body and can modulate the normal physiology of the body. ${ }^{2}$

Apoptosis and necrosis are the normal biological processes which controls and regulates the cell division and cell growth. Among them, apoptosis is a major player which regulates the cell division and cell growth. Hence, there will be no tumours or cancer, if the body's apoptosis process works properly. Every cancer tumors have an inhibited apoptosis process.
Hence, normal apoptosis process is a preventive action for cancer genesis.

In the body, there is a protein which driven and controls the apoptosis. This protein is p53 protein. So basically it is a tumor suppressor protein. Approximately half of all human cancers have inactivating mutations of $\mathrm{p} 53$, while the rest have adapted ways to override the tumor suppressor function of p53.

The activity of the p53 protein, has been influence or modulated by the murine double minute 2 (MDM2). MDM2 is an Oncoprotein, and it is a major antagonist of the p53.

p53 and MDM2 interact with each other via autoregulatory negative feedback loop. Upon activation, p53 binds to the P2 promoter of the MDM2 gene and transcriptionally induces MDM2 protein expression. And the expression of MDM2 results into the lowering of the biological level of p53 and inhibition of the p53 
function. Hence, this protein-protein interaction inhibits the p53 function, and leads to malignant transformation of cells.

This protein-protein p53-MDM2 interaction is responsible for the inhibition of p53 and apoptosis. And also reason for the tumour genesis. So if we can inhibit this interaction, we can make the p53 protein freed. Which initiates the activation of the normal apoptosis in the cancer cells. This interaction can be inhibited by the various inhibitors, like peptide or nonpeptide inhibitors. Among them, non-peptide small molecule inhibitors are the most convenient and prominent.

\section{Small molecule (NONPETIDIC) inhibitors of the p53-MDM2 interaction}

Every protein-protein interaction covers the large surface area because the proteins are polymer in nature. Hence to target or to inhibit any protein-protein interaction by small molecules are very much treaky. ${ }^{3}$

By studying the crystal structure of MDM2 protein, it is revealed that there is a deep hydrophobic pocket in the MDM2 protein, which is getting packed/filled by the three side chains of the p53 protein.

So if we design the non-peptidic small molecules which are identical with the three residues of the side chains of the p53 protein, then it will mimic the p53 protein by three pocket binding. So that the binding of the p53 protein with the MDM2 getting inhibited and we can prevent the p53-MDM2 protein-protein interaction. ${ }^{4}$ By using this, we can spare the p53 protein and its activation getting occurred. And ultimately it will trigger the p53 dependent apoptosis in the cancer cells.

In the residue of the side chain of $\mathrm{p} 53$, there are mainly three amino acids, which play the role in the protein binding (Phe19, Trp23 and Leu26). These three amino acids, sits/fit into the MDM2 pocket (3 pocket binding) and p53 function getting inhibited. Among, these three amino acids, ${ }^{5}$ Trp23 is most important because it forms the hydrogen bond with the MDM2 pocket also having hydrophobic interaction with that.

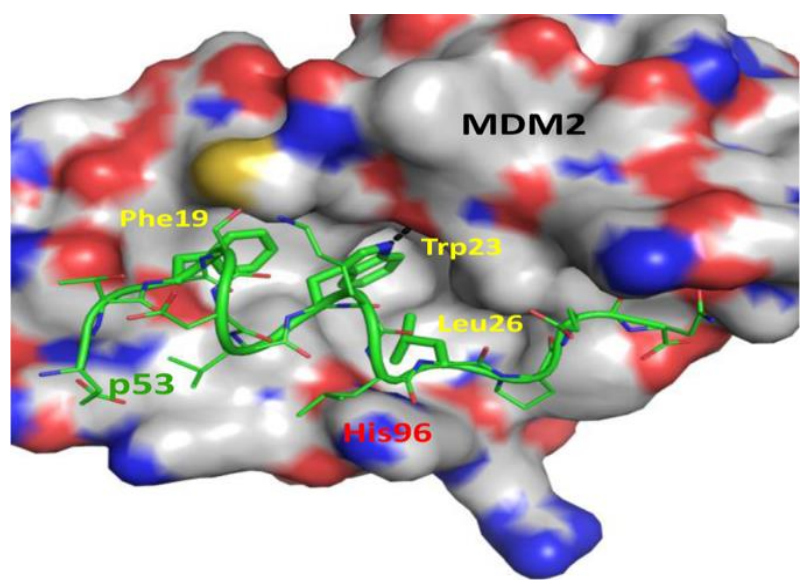

Fig. 1: p53 bound with the MDM2 Protein (p53MDM2 Complex) ${ }^{6}$

In 2004, Vassilev and his colleagues at Roche Pharmaceutical reported the first p53-MDM2 interaction inhibitor or the MDM2 inhibitor, termed as "Nutlin". It was cis-imidazoline analogue and having the IC50 value 100 to $300 \mathrm{nM}^{7}$

Nutlin 1, $2 \& 3$ are the same series of compounds, having the different MDM2 inhibition potency. Nutlin $3 \mathrm{a}$ is the most potent compound of the class. And $3 \mathrm{a}$ is 150 times more active than its enantiomer $3 b$. Nutlins class of compounds can successfully mimic the p53 side chain's residue and it is having three pocket binding.

Superimposition of 3D structure of Nutlin on cocrystal structure of MDM2 protein shows that, one bromo-phenyl ring of the Nutlin fits into the Trp23 pocket of MDM2 protein. The other bromo-phenyl ring fits into the Leu26 pocket and the ethyl ether side chain sits into the Phen19 pocket. ${ }^{8}$ So it is having the three pocket binding

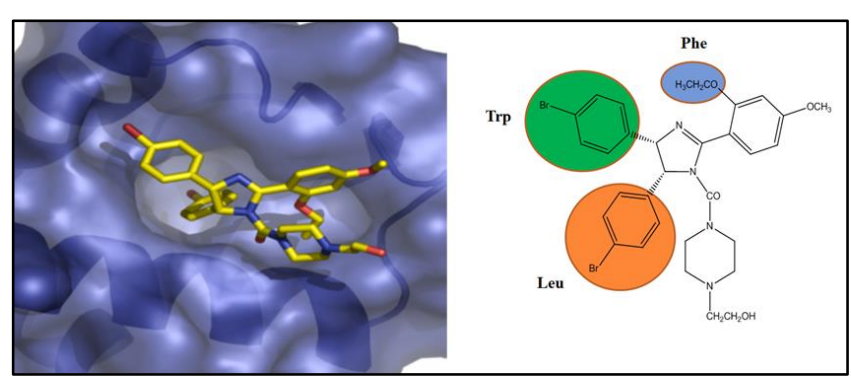

Fig. 2: Crystal Structure of the Nutlin-2 bound MDM2 Protein ${ }^{9}$ 
This study is foundation for the design of a nonpeptide, small molecule inhibitors of p53-MDM2 Interaction. Recently, we are working on the development of this class of compounds.

For the successful design of the MDM2 inhibitors, it should possess the following characteristic:

1. Accumulation and stabilization of $\mathrm{p} 53$ protein. (By preventing its degradation and its export from the nucleus)

2. Activation of p53 pathway and various genes, which regulates p53 function. (Induce cell cycle arrest in the G1and G2 phases and/or apoptosis).

The various studies shows that, p53 protein getting liberated from the MDM2 protein by using the MDM2 inhibitors and p53 getting accumulated in the nuclei of cancer cells. So that, the p53 function as well as its pathways getting activated. And the proliferating cancer cells were effectively blocked in G1 and G2 phases (G2/M phase fraction and nearly complete depletion of the S-phase). ${ }^{10}$ These effects trigger the cell cycle arrest and the cell death/apoptosis in the cancer cells.

Unfortunately, various studies says that the p53 function and apoptosis was only occurred in the cancer cells which contains the wild type p53 protein not in the cancer cells which contains the mutated/deleted p563 protein. ${ }^{11}$ So there will be low cytotoxicity seen in the cancer cells which contains the mutant p53 protein.

The non-peptidic small molecule MDM2 inhibitors have a good cell permeability to cell over the peptidic inhibitors. Because peptides are the polymer and high molecular weight compounds. And peptides types of inhibitors having tendency to get degraded by the body's metabolic enzymes and processes.

Many of the small molecule inhibitors does not follows the Lipinski rule in the case of molecular weight, many of the inhibitors are at the upper limits of a molecular weight cut-off (Lipinski's rule of five). ${ }^{12}$

After the discovery of the Nutlin class of compounds, numbers of p53-MDM2 Interaction Inhibitors have been reported by various researchers and companies. These all are having the different chemical moieties like benzodiazepinediones, cisimidazolines, oxindoles, spirooxindoles, chalcones,chlorofusin (natural product), tryptamine and other numerous miscellaneous groups.

\section{Clinical development of p53-MDM2 inhibitors:}

RG7112 (R7112, RO5045337) was the first compound to enter into clinical trials. As of the Nutlins, It has also developed by the Hoffmann-La Roche pharmaceuticals. And it is having the identical chemical moiety, which is cis-imidazoline [13]. It is an orally administered agent. It inhibits p53/MDM2 interaction and inhibits the proteosomal degradation of p53. So p53 getting stabilized and increased its amount.

In this review, we have covers the details of the non-peptide small molecule p53-MDM2 Interaction Inhibitors which are under clinical trials. And we have also included the structures of respective compounds.

Table 1: MDM2 Inhibitors under Clinical trials ${ }^{14-18}$

\begin{tabular}{|l|c|l|l|}
\hline \multicolumn{1}{|c|}{ Compound } & $\begin{array}{c}\text { Phase of } \\
\text { Clinical Trial }\end{array}$ & Status & \multicolumn{1}{|c|}{ Originator } \\
\hline RG7112(RO5045337) & 1 & Active & Hoffmann-La Roche \\
\hline $\begin{array}{l}\text { RG7388 (RO5503781) } \\
\text { (Idasanutlin) }\end{array}$ & 1 & Active & Hoffmann-La Roche \\
\hline MI-77301 (SAR405838) & 1 & Active & $\begin{array}{l}\text { University of Michigan, Advanced into } \\
\text { clinical trials by Sanofi in 2012 }\end{array}$ \\
\hline MK-8242 (SCH 900242) & 1 & Active & Merck \\
\hline AMG232 & 1 & Active & Amgen Biopharma \\
\hline DS-3032b & 1 & Active & Daichi Sankyo \\
\hline
\end{tabular}




\begin{tabular}{|l|l|l|l|}
\hline HDM201 & 1 & Active & Novartis \\
\hline CGM097 & 1 & Active & Novartis \\
\hline ALRN-6924 (Stapled Peptides) & 1 & Active & Aileron Therapeutics and Roche \\
\hline
\end{tabular}

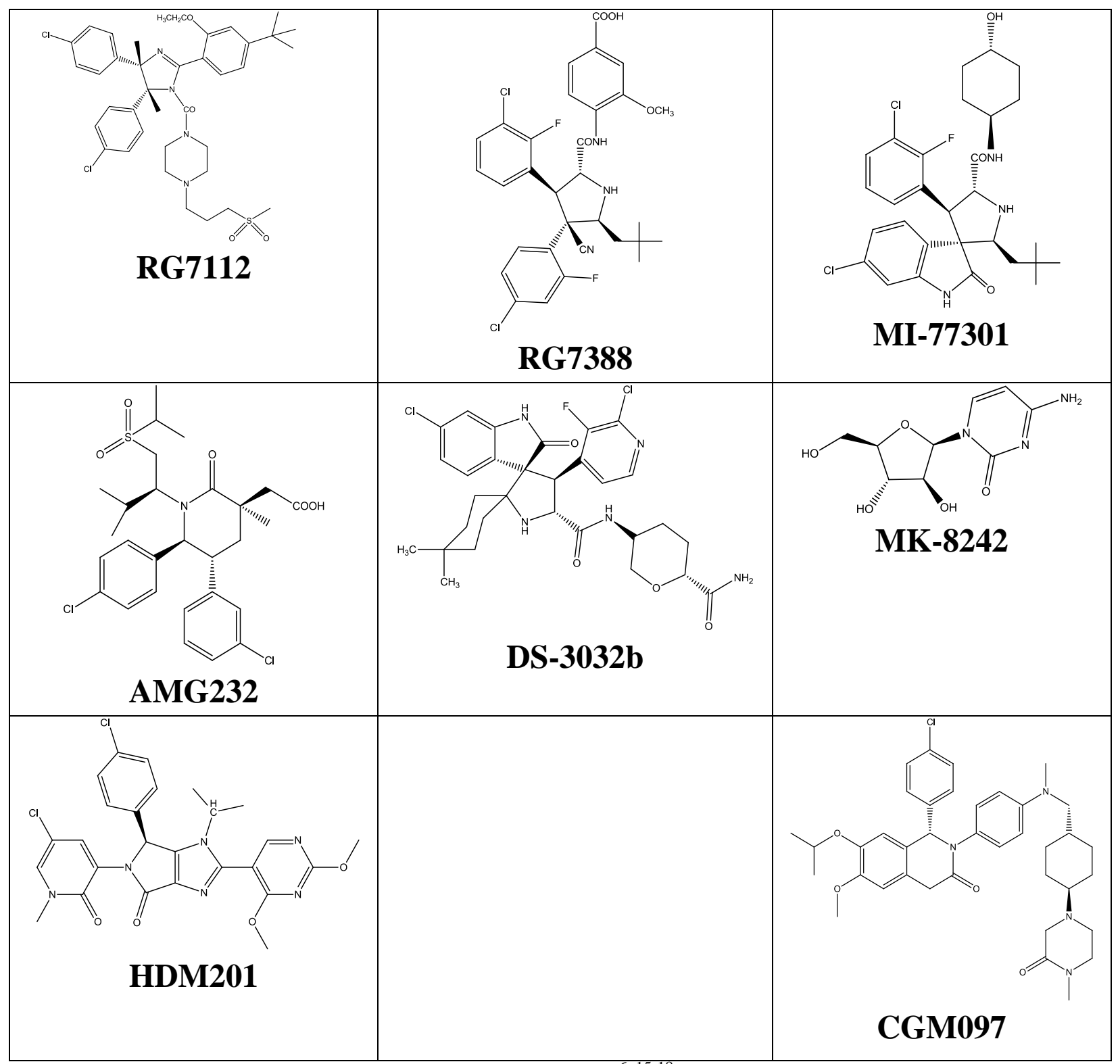

Fig. 3: Structures of the MDM2 Inhibitors under Clinical trials, ${ }^{6,15-18}$

The IC50 values of the MDM2 inhibitors which are under clinical trials are as follow [6, 19]:

Table 1: IC50 values of the MDM2 inhibitors

\begin{tabular}{|c|l|c|}
\hline S. No & \multicolumn{1}{|c|}{ Compound } & \multicolumn{1}{c|}{ IC 50 value (nM) } \\
\hline 01 & RG7112 (RO5045337) & 18 \\
\hline 02 & $\begin{array}{l}\text { RG7388 (RO5503781) } \\
\text { (Idasanutlin) }\end{array}$ & 100-200 (Cell lines: SJSA-1, RS411, LNCaP, HCT116) \\
\hline 03 & MI-77301 (SAR405838) & 20 \\
\hline 04 & MK-8242 (SCH 900242) & \\
\hline
\end{tabular}




\begin{tabular}{|c|l|c|}
\hline 05 & AMG232 & 0.6 \\
\hline 06 & DS-3032b & 5.57 \\
\hline 07 & HDM201 & 0.21 \\
\hline 08 & CGM097 & 1.7 \\
\hline
\end{tabular}

The substantial research and effort yields the some of the promising compounds of this class. And many of the compounds have been successfully entered into phase of the clinical trials

\section{Application of p53-MDM2 interaction inhibitors (MDM2 INHIBITORS) in cancer therapy}

The conventional anti-cancer drugs are genotoxic. So they induced the p53 depended apoptosis by the genetic damage. The conventional drugs activated the p53 and induced the apoptosis by phosphorylation of the protein on specific serine residues (Ser15 is phosphorylated most frequently) near the MDM2 binding domain. Whilst small molecule MDM2 inhibitors does not damaged the genetic material, and can directly activated the p53 function into the cells. So small molecule MDM2 inhibitors can activate the p53 and induces the apoptosis without the phosphorylation of the protein.13

Since p53 is an anti tumour protein so its activation by small molecule MDM2 inhibitors would induce the apoptosis in cancer cells.

Its application in cancer therapy can be achieved through various strategies like (i). Inhibiting MDM2 expression (ii). Inhibiting the p53-MDM2 interaction (iii). Modulating the E3 ubiquitin ligase activity of MDM2 (iv). Targeting the MDM2-p53 complex.

Among these strategies, the p53/MDM2 interaction inhibition is most promising and most studied.

Clinical data evident that, in the cancer cell lines, activation of $\mathrm{p} 53$ by MDM2 inhibitors induces p53 dependent cell cycle arrest and cell death. (In cells with wild-type p53, but not in cells with deleted or mutated p53). While in the normal cells, it leads to the dose dependent cell cycle arrest, but not cell death.

Hence, p53 activation by MDM2 inhibitors could be selectively target the cancer cells over the normal cells.

To minimize the side effects of small molecule MDM2 inhibitors, there are many approaches, among them, cyclotherapy is a most suitable approach to lower down the side effects. In cyclotherapy, there is a conditioning treatment with low doses of p53 activators to induce cell cycle arrest in normal cells with a cytostatic effect that will be protected from the toxicity of conventional drugs targeting the S/M phases of the cell cycle.

Small molecule MDM2 inhibitors having a good anti-tumor activity in a both, in vitro and in vivo too. But unfortunately it cannot target or target little to the cells which contains the mutated p53 protein. Because mutated p53 protein cannot activate its negative regulator (MDM2 protein) and disruption of $\mathrm{p} 53$ MDM2 binding.

TP53 gene might be mutated or deleted in the cancer cells. So it will be non-effective as a transcription factor. ${ }^{20}$ Clinically, with the help of DNA microarray based GeneChip we can determine the mutant p53 protein. $^{21}$

\section{Discussion}

To target the PPI and to inhibit the MDM2 protein is prerequisite to reactivate the p53 function and the apoptosis. Small molecule inhibitors are capable of, to inhibit this phenomenon. So small molecules will reactivate the p53 function and trigger the apoptosis in the cancer cells. The reactivation of p53 function might be offer an effective therapeutic strategy for tumors that retains the wild-type p53 and in which the downstream p53 dependent apoptotic signaling is preserved. The only limiting factor is that, small molecules can't able to reactivate the p53 function in cancer cells which contains the mutated or deleted p53, so it need to be address by more scientific efforts. Significant advancements have been occurring for this class by the entry of some of the small molecule MDM2 inhibitors into clinical trials. Alongside, Improve bioavailability and optimized PK profile of small molecule MDM2 inhibitors has required further investigation. 


\section{Source of Funding}

None.

\section{Conflict of Interest}

None.

\section{References}

1. Tripathi KD, Essentials of Medical Pharmacology; 6th Edn; Jaypee Brothers Medical Publishers Pvt Ltd, New Delhi, 2010, 819-34

2. Gohil CJ and Noolvi MN, "Synthesis of New Diaryl Derivatives Comprising Imidazothiadiazole Moiety as Potential Anticancer Agents", IJPCA 2015;2(2):84-96

3. Arkin MR and Wells JA, "Small-Molecule Inhibitors of Protein-Protein Interactions: Progressing towards the Dream", Nat Rev Drug Discov 2004;3:301-17

4. Vassilev LT, "p53 Activation by Small Molecules: Application in Oncology", J Med Chem 2005;48(14):44919.

5. Ding K, Lu Y, Coleska ZN, Qiu S, Ding Y, Gao W et al. "Structure-Based Design of Potent Non-Peptide MDM2 Inhibitors", J Am Chem Soc 2005;127:10130-1

6. Zhao Y, Aguilar A, Bernard D, Wang S, "Small-Molecule Inhibitors of the MDM2-p53 Protein-Protein Interaction (MDM2 Inhibitors) in Clinical Trials for Cancer Treatment", J Med Chem 2015;58:1038-52.

7. Vassilev LT, Vu BT, Graves B, Carvajal D, Podlaski F, Filipovic Z et al. "In Vivo Activation of the p53 Pathway by Small-Molecule Antagonists of MDM2", Sci 2004;303(5659):844-8.

8. Vassilev LT, "MDM2 Inhibitors for Cancer Therapy", Trends Mol Med 2006;13(1):23-31.

9. Deng J, Dayam R, Neamati N, "Patented small molecule inhibitors of p53-MDM2 interaction", Expert Opin Ther Patents 2006;16(2):165-8.

10. Gohil CJ and Noolvi MN, "Types of p53/MDM2 Interaction Inhibitors", Eur J Bio Med Pharm Sci 2018;5(11):143-7.

11. Shangary S and Wang S, "Small-Molecule Inhibitors of the MDM2-p53 Protein-Protein Interaction to Reactivate p53
Function: A Novel Approach for Cancer Therapy", Annu Rev Pharmacol Toxicol 2009;49:223-41.

12. Fotouhi N and Graves B, "Small Molecule Inhibitors of p53/MDM2 Interaction", Curr Top Med Chem 2005;5(2):159-65.

13. Gohil CJ and Noolvi MN, "p53 Protein: Master Regulator of Apoptosis and its Application in Cancer Therapy" Int J Pharm Bio Arch 2019;10(3):154-64.

14. Tisato V, Voltan R, Gonelli A, Secchiero P, Zauli G, "MDM2/X inhibitors under clinical evaluation: Perspectives for the management of hematological malignancies and pediatric cancer", J Hematol Oncol 2017;10(133):1-17

15. DS-3032b, ChemIDplus, A Toxnet Database, U.S. National Library of Medicine https://chem.nlm.nih.gov/chemidplus/rn/1398569-75-9

16. HDM201, ChemIDplus, A Toxnet Database, U.S. National Library of Medicine https://chem.nlm.nih.gov/chemidplus/rn/1448867-41-1

17. CGM097, Pubchem Open Chemistry database, National Centre for Biotechnology Information, U.S. National Library of Medicine https://pubchem.ncbi.nlm.nih.gov/compound/nvpcgm097\#section=Top

18. CGM097, Drug Profile, Adis Insight, Springer https://adisinsight.springer.com/drugs/800037008

19. Liao G, Yang D, Ma L, Li W, Hu L, Zeng L, et al. "The Development of Piperidinones as Potent MDM2-P53 Protein-Protein Interaction Inhibitors for Cancer Therapy", Eur J Med Chem 2018;159:1-9.

20. Hainaut $P$ and Hollsteln M, "p53 and Human Cancer: The First Ten Thousand Mutations", Adv Cancer Res 2000;77:81-137

21. Ahrendt SA and Halachmi S, Chow JT, Wu L, Halachmi N, Yang SC et al. "Rapid p53 sequence analysis in primary lung cancer using an oligonucleotide probe array", Proc Natl Acad Sci USA 1999;96:7382-7.

How to cite this article: Gohil CJ, Noolvi MN. Non peptidic small molecular inhibitors of the p53-MDM2 interaction. Int $J$ Pharm Chem Anal 2019;6(4):104-9. 